\title{
PROOF OF A WARING THEOREM ON FIFTH POWERS*
}

\author{
BY L. E. DICKSON
}

In 1770 , E. Waring conjectured that "every number is a sum of nine cubes, also is a sum of 19 fourth powers, and so on to infinity."

By elaborate and very delicate analysis, Hardy and Littlewood obtained remarkable asymptotic results. Their proofs apply only to numbers exceeding an extremely large limit $L$.

I shall here explain my method to cover the numbers $<L$ and hence finally obtain universal theorems holding from 1 to infinity.

For greater clearness, I shall first consider briefly the case of cubes. Jacobi inspired the construction of tables showing the least number of positive cubes (or fourth powers) whose sum yields the tabulated number. In 1851 , he published the table to 12,000 by $Z$. Dahse. In 1903, von Sterneck made a table to 40,000 and found not merely that nine cubes always suffice, but also the more vital fact that all numbers between 8,042 and 40,000 are sums of six cubes.

I shall prove that every number $N$ lying between 40,000 and 67,000 is a sum of seven cubes. From all these numbers we subtract 27,000 , which is the cube of 30 . Take 27,000 from 40,000 and you have 13,000 . Take 27,000 from 67,000 and you have 40,000 . Since $N-30^{3}$ therefore lies between 13,000 and 40,000, it is a sum $S$ of six cubes by the result quoted. Hence $N=30^{3}+S$ is a sum of seven cubes.

Similarly, every number $M$ lying between 67,000 and 94,872 is a sum of seven cubes, since $M-38^{3}=M-54,872$ lies between 12,128 and 40,000 and hence is a sum of six cubes, so that $M$ is a sum of seven cubes.

This argument may be repeated until we have finally subtracted the cube of 103 . Our result is that all numbers between 8,042 and $1,132,727$ are sums of seven cubes.

\footnotetext{
* An address, presented to the Society at the request of the program com-
} mittee, April 4, 1931. The presentation was made with the aid of lantern slides. 
Evidently a like argument shows that still larger numbers are sums of eight cubes, in fact, all between 454 and 232,604,691.

A final such argument shows that all numbers up to 4 billion are sums of nine cubes.

We obtained this conclusion quite simply from facts in the table to 40,000. We next indicate its importance for the general theory of cubes. In 1909, Wieferich proved that all numbers exceeding $2 \frac{1}{4}$ billions are sums of nine cubes. Since our two limits overlap, we finally conclude that all numbers are sums of nine cubes. We depend upon the advanced mathematical proof for very large numbers and depend upon our deductions from a table for the smaller numbers.

I now pass to my recent analogous investigations on sums of fifth powers. I made a table which shows the least number (called minimum) of fifth powers whose sum is the tabulated number less than 67,232 .

We shall make use only of the part of the table after 32,107 . In this part there occurs no minimum 19 or greater. After 50,425 , there occur only the following ten numbers $N$ having the minimum 18:

$54430,54738,54770,55211,55276,55393,55571,56057$, $63769,63801$.

To each $N$ we add $7^{5}=16,807$ and get the only possible minima $\geqq 19$ between 67,232 and 84,039 . To show that each such sum $N+7^{5}$ actually has a minimum $\leqq 18$, we note that $N+7^{5}-8^{5}=N-15961$ has the values $38469, \cdots, 47840$, which are seen by our table to have minima $\leqq 17$. Transposing $8^{5}$, we conclude that $N+7^{5}$ has a minimum $\leqq 18$. This proves that not merely in our table, but also in an imaginary extension of it to 84,039 , there occurs no minimum $\geqq 19$.

If we repeat this argument, adding $8^{5}$ instead of $7^{5}$ and subtracting $9^{5}$ instead of $8^{5}$, we secure the further extension of our result to 100,000 . Hence finally we conclude that all numbers from 32,017 to 100,000 are sums of 18 fifth powers.

Just as we ascended from 6 to 7 cubes, so here we ascend from 18 to 19 fifth powers. To prove that every number $N$ between 100,000 and $100,000+9^{5}=159,049$ is a sum of 19 fifth powers, note that $N-9^{5}$ lies between 40,951 and 100,000 and hence by our preceding result is a sum of 18 fifth powers. Similarly, we may add $10^{5}$ and $11^{5}$ instead of $9^{5}$, and obtain the result that all 
numbers from 32,017 to $100,000+11^{5}=261,051$ are sums of 19 fifth powers.

We may obtain this final limit at once, without employing our three steps, and similarly avoid the many steps used for cubes. To do this we apply the following theorem with $l=32,107$, $g=100,000, k=19, m=10$.

THEOREM. Grant that every number $n$ between $l$ and $g$ is a sum of $k-1$ fifth powers. Find the maximum integer $m$ such that $(m+1)^{5}-m^{5}<g-l$. Then every number between $l$ and $g+(m+1)^{5}$ is a sum of $k$ fifth powers.

To ascend to 20 fifth powers, apply the theorem with $l=32,017, g=261,051, k=20$. Now $m=14$, whence every number between $l$ and $g+15^{5}=1,020,426$ is a sum of 20 fifth powers. Similarly, all numbers between 32,017 and

$$
\begin{aligned}
& 5,104,527 \text { are sums of } 21 \\
& 38,658,959 \text { " " " } 22
\end{aligned}
$$

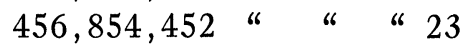

$$
\begin{aligned}
& 9,496,062,420 \text { " } \quad \text { " } 24 \text {. }
\end{aligned}
$$

Continuations of these results are best made by an older theorem which is convenient for logarithmic work. It would have been very wasteful for the preceding ascents, but gives nearly as good results for the future ascents. This older theorem is the following: If numbers $<L$ are sums of $n$ fifth powers, we compute the limit under which numbers are sums of $n+1$ fifth power by multiplying $L / 5$ by its fourth root. Evidently these limits will increase faster and faster as we ascend and yield limits too large to write out in full, so that we shall merely tell the number of figures involved in them. The most significant results are the following two: All numbers having fewer than 122 figures are sums of 37 fifth powers. All having fewer than 454 figures are sums of 43 fifth powers.

Hardy and Littlewood, after years of work, finally succeeded in proving, by very long and most delicate analysis, that all numbers involving more than 445 figures* are sums of 54 fifth powers. Combining this result with our preceding one, we conclude that all numbers from 1 to infinity are sums of 54 fifth powers.

\footnotetext{
* This limit was obtained by Dr. A. Oppenheim at the request of the writer.
} 
Later I extended my table to 138,000 . We shall use only the 39,400 numbers between 98,600 and 138,000 . By the methods explained before, this part of the table enabled me to prove that all numbers between 98,605 and 200,000 are sums of 16 fifth powers. Using merely this fact, we may ascend as usual and find that all numbers having fewer than 194 figures are sums of 37 fifth powers, while all having fewer than 469 figures are sums of 41 . We have not only secured better results than before, but also have secured an independent new proof of the main theorem that all numbers are sums of 54 fifth powers.

Finally, with the aid of assistants, I extended my table to 300,000 . By using the minima $\geqq 14$ in the last half of the table, I proved that from 300,000 to 837,824 there are only 31 numbers of minimum 15 (and none of greater minimum), with an average of 1 per thousand in the first 15 thousands after 300,000 , but with later thinning out and terminating with 470,348 . Hence from the latter to 837,824 , every number is a sum of 14 fifth powers. By the methods already explained, I used the last fact to prove that

All between 191,263 and 98,605

87,919

32,017

30,221 $4,037,824$ are sums of 15

22,626 $28,337,824$

All with $<484$ figures are sums of 37

All with $<1177$ figures are sums of 41 .

It therefore seems almost certain that every number is a sum of 37 fifth powers (while 37 are necessary to get the number 223). We also notice a dropping from 37 which is quite rapid to 18 and later quite slowly to $17,16,15,14$. A further dropping to 13 was secured recently. By studying the minima $\geqq 13$ just before 300,000 , I proved that each of the 52,841 numbers between 786,159 (which requires 14 fifth powers) and 839,000 is a sum of 13 fifth powers. This remarkable fact implies that 14 suffice not merely to the former limit 837,824 , but on to 940,000 , which would permit us to raise somewhat all the limits in the last tablette. 
A final word about the actual construction of my table of fifth powers. The earlier table of fourth powers was made by finding all ways of expressing each number as a sum of fourth powers and then picking out the ways involving the fewest powers. But the number of ways of expressing $n$ as a sum of fourth (or fifth) powers increases extremely rapidly as $n$ increases, whence this method is both highly impracticable and liable to many errors.

My method finds a minimum mode of expression without knowing all modes of expression. To illustrate, write

$A=2^{5}, \quad B=3^{5}, \quad C=4^{5}, \quad D=5^{5}, \quad E=6^{5}, \quad F=7^{5}, \quad G=8^{5}$, and consider decompositions involving only $A, B, C, F$. Those not appearing in the following tablette

$$
\begin{array}{rllr}
3 F & B+3 F & 2 B+3 F & 3 B+3 F \\
A+3 F & A+B+3 F & A+2 B+3 F & A+3 B+3 F \\
2 A+3 F & 2 A+ & 2 A+ \\
3 A+3 F & 3 A+ & 3 A+ \\
4 A+3 F & 4 A+ & 4 A+ & \\
5 A+3 F & 5 A+ & 5 A+
\end{array}
$$

cannot be minimum decompositions in view of the four equations

$6 A+3 F=2+B+2 C+2 E+G, 2 A+3 B+F=2 C+2 E$, $4 B+3 F=1+3 C+2 E+G, C+3 F=D+2 E+G$.

The numerical values of the entries in our tablette were copied in to the big table and the rare cases of duplicates and non-minima were deleted.

The University of Chicago 